\title{
In situ measurements of complex permittivity and moisture content in oil palm fruits.
}

\begin{abstract}
An in situ measurement system for oil palm fruit's permittivity and moisture content determination is presented. The instrumentation is includes a six-port reflectometer and an open ended coaxial probe which operates at $2 \mathrm{GHz}$. The developed system was tested and compared with the data obtained using commercial dielectric measurement probe and mixture model. This system is found to be practical for in situ measurement as well as fast and cost effective for oil palm fruit's properties and quality inspection.
\end{abstract}

Keyword: PACS. 07.57-c Infrared; Submillimeter wave; Microwave; Radiowave instrumentations. 